\title{
Definition of averaged elastic-plastic characteristics of sandwich panel structures
}

\author{
I. I. Zakirov, V. N. Paimushin \& I. M. Zakirov \\ A. N. Tupolev State Technical University, Kazan, Russia
}

\begin{abstract}
Analytic formulas for averaged elastic and strength characteristics of folded structure's determination are identified. They are based on the introduction of a hypothesis about momentless action of its elements (web covers) in a charge operation and on phased deletion from action ex-post buckling. Dimensionless coefficients contained in the structural formulas, which appear on the solution process of formulated problems, are to be determined from experimental evidence on tension, compression and pure shear in two-planes. In a tensioncompression process the folded structures should be considered heterogeneous material with averaged plasto-elastic behaviors, which has relations between averaged voltages and averaged appropriate deformations and has a deformations stepwise change area (conditional plastic flow).
\end{abstract}

Keywords: folded structures, cell of cyclicity, averaged elastic and strength characteristics, modulus of elasticity, deformation curve, structural formulas, theoretical and experimental method.

\section{Introduction}

In many cases, the use of composite materials is the most efficient in constructions (three-layer or multilayer) with cores, which have good mechanical strength, rigidity, vibration, sound and heat insulation characteristics. These characteristics of cores of some structures have been well studied today. A large number of publications, a detailed analysis of which is contained, in particular, in a review article [1] are devoted to their research.

Most often in three-layer and multilayer structures, the cores have a honeycomb $([2,10]$ etc. $)$ structure. In the calculations of such structures the real cores are usually replaced by some homogeneous continuum, which averaged 
mechanical and thermal characteristics are determined by the principle of equivalence of real and replacing core [3-5]. At such replacement of real honeycomb with orthotropic continuum for the strength calculations averaged elastic module in the tangential directions and the shear modulus in the tangential plane are set equal to zero, and the elastic modulus in the transverse direction and shear module in the transverse plane is determined by theoretical or experimental methods $[8,10]$. Formulas for their determination are given, in particular, in references $[3,4]$ that, as a first approximation of formulas, have been obtained by the energy method [3], or the method of displacements and forces [6]. Along with these, there are other theoretical methods for determining the averaged elastic characteristics of periodic structures based, in particular, on the method of averaging the solution of problems by Bakhvalov and Ponasenko [7].

In recent years, ways of production of the folded structures from flat sheets cores $[11,12]$ develop very intensively. Such cores compared with honeycomb cores have a number of fundamental differences and advantages. In this paper we consider one of these folded structures. We develop relevant theoretical and experimental method to determine the homogenized elastic and strength characteristics of which.

\subsection{Formulation of the problem}

Consider folded structure with a quadriradiare core [11], formed from a flat sheet blank with thickness $t$ by means of its gradual transformation into a state of relief, as shown in Figure 1a. We assume that, in general, $l_{1} \neq l_{2}, \varphi_{1} \neq \varphi_{2}$ and all four sloping walls $1,1^{\prime}, 2,2^{\prime}$ of the periodicity cells isolated from the core are a parallelogram, symmetrically placed relative to the plane $x 0_{1} z$. Therefore, the middle planes $\sigma_{(k)}$ of each of them reasonable classified as a local oblique coordinate system $x_{k} 0_{k} y_{k}$ and $x_{k}^{\prime} 0_{k} y_{k}^{\prime}$, in which (Fig. 1a) $x_{k}=x_{k}^{\prime}$ and the radius vectors of the points $\sigma_{(k)}$ given by a representation

$$
\boldsymbol{r}^{(k)}=x_{k} \boldsymbol{e}_{1}^{(k)}+y_{k} \boldsymbol{e}_{2}^{(k)}, \quad \boldsymbol{r}^{\left(k^{\prime}\right)}=x_{k}^{\prime} \boldsymbol{e}_{1}^{\left(k^{\prime}\right)}+y_{k}^{\prime} \boldsymbol{e}_{2}^{\left(k^{\prime}\right)}
$$

In them unit vectors $\boldsymbol{e}_{i}^{(k)}, \boldsymbol{e}_{i}^{\left(k^{\prime}\right)}$ satisfies the equations

$$
\boldsymbol{e}_{i}^{(k)} \boldsymbol{e}_{i}^{(k)}=1, \boldsymbol{e}_{i}^{\left(k^{\prime}\right)} \boldsymbol{e}_{i}^{\left(k^{\prime}\right)}=1, \boldsymbol{e}_{1}^{(k)} \boldsymbol{e}_{2}^{(k)}=\boldsymbol{e}_{1}^{\left(k^{\prime}\right)} \boldsymbol{e}_{2}^{\left(k^{\prime}\right)}=\cos \varphi_{k}
$$

which according to Fig. $1 \mathrm{a}$ and $1 \mathrm{~b}$ with unit vectors $\boldsymbol{i}, \boldsymbol{j}, \boldsymbol{k}$ of orthogonal Cartesian coordinate system related with dependencies 


$$
\begin{aligned}
& \boldsymbol{i e}_{1}^{(k)}=\boldsymbol{i} e_{1}^{\left(k^{\prime}\right)}=\cos \alpha_{k}, \boldsymbol{i} e_{2}^{(k)}=\boldsymbol{i} e_{2}^{\left(k^{\prime}\right)}=\sin \psi \\
& \boldsymbol{j} \boldsymbol{e}_{2}^{(k)}=\cos \psi, \boldsymbol{j} \boldsymbol{e}_{2}^{\left(k^{\prime}\right)}=-\cos \psi, \boldsymbol{j} \boldsymbol{e}_{1}^{(k)}=\boldsymbol{j} \boldsymbol{e}_{1}^{\left(k^{\prime}\right)}=0 \\
& \boldsymbol{k} \boldsymbol{e}_{1}^{(1)}=\boldsymbol{k} \boldsymbol{e}_{1}^{\left(1^{\prime}\right)}=\sin \alpha_{1}, \boldsymbol{k} \boldsymbol{e}_{1}^{(2)}=\boldsymbol{k} \boldsymbol{e}_{1}^{\left(2^{\prime}\right)}=-\sin \alpha_{2} \\
& \boldsymbol{k} \boldsymbol{e}_{2}^{(k)}=\boldsymbol{k} \boldsymbol{e}_{2}^{\left(k^{\prime}\right)}=0
\end{aligned}
$$
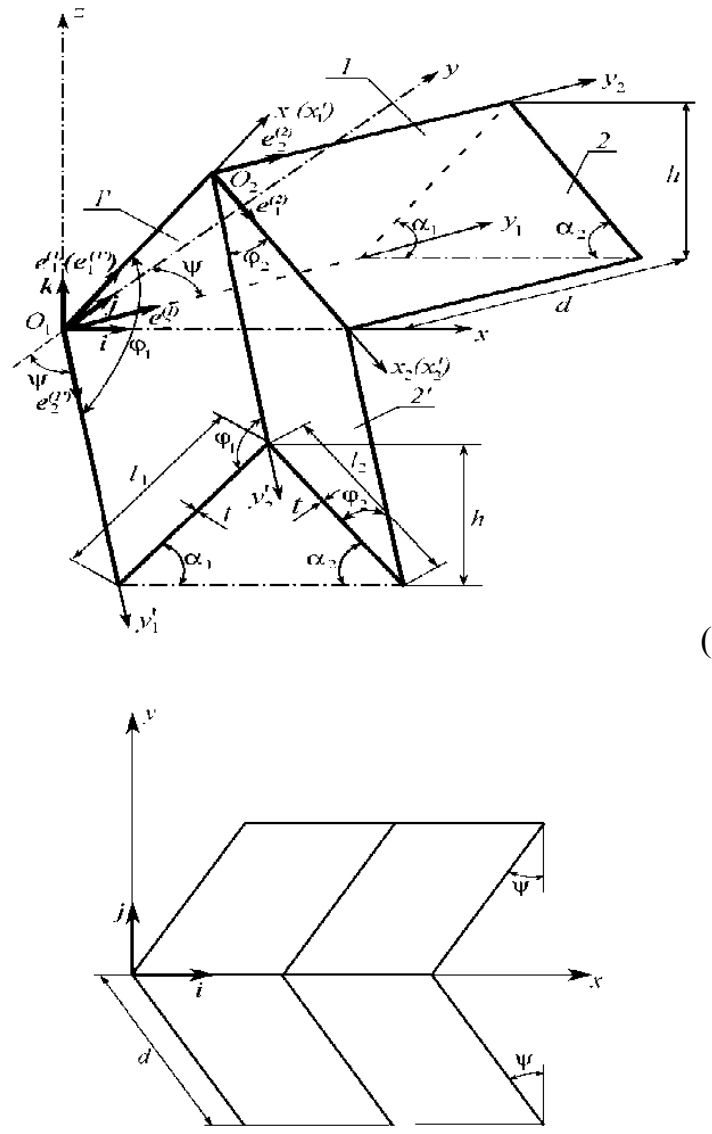

(b)

Figure 1.

Parameterizations (1.1) correspond to the basis vectors (for the elements $1^{\prime}$ and $2^{\prime}$ compiled following relations must be marked with strokes)

$$
\boldsymbol{r}_{1}^{(k)}=\partial \boldsymbol{r}^{(k)} / \partial x_{k}=\boldsymbol{e}_{1}^{(k)}, \boldsymbol{r}_{2}^{(k)}=\partial \boldsymbol{r}^{(k)} / \partial y_{k}=\boldsymbol{e}_{2}^{(k)}
$$


co- and contravariant components of metric tensor

$$
\begin{gathered}
a_{i i}^{(k)}=\boldsymbol{r}_{i}^{(k)} \boldsymbol{r}_{i}^{(k)}=1, a_{12}^{(k)}=a_{21}^{(k)}=\boldsymbol{r}_{1}^{(k)} \boldsymbol{r}_{2}^{(k)}=\cos \varphi_{k} \\
a_{(k)}^{i i}=\frac{a_{i i}^{(k)}}{a_{(k)}}=\frac{1}{\sin ^{2} \varphi_{k}}, a_{(k)}^{12}=a_{(k)}^{21}=-\frac{a_{12}^{(k)}}{a_{(k)}}=-\frac{\cos \varphi_{k}}{\sin ^{2} \varphi_{k}}
\end{gathered}
$$

where $a_{(k)}=a_{11}^{(k)} a_{22}^{(k)}-a_{12}^{(k)} a_{12}^{(k)}=1-\cos ^{2} \varphi_{k}=\sin ^{2} \varphi_{k}$.

For the displacement vectors of core walls we accept following representations

$$
\begin{aligned}
& \boldsymbol{U}^{(k)}=u^{(k)} \boldsymbol{e}_{(k)}^{1}+v^{(k)} \boldsymbol{e}_{(k)}^{2}+w^{(k)} \boldsymbol{m}^{(k)}-z_{(k)}\left(\psi^{(k)} \boldsymbol{e}_{(k)}^{1}+\chi^{(k)} \boldsymbol{e}_{(k)}^{2}\right) \\
& -t / 2 \leq z_{(k)} \leq t / 2
\end{aligned}
$$

where $\boldsymbol{e}_{(k)}^{i}$ are the reciprocal basis vectors satisfying the conditions $\boldsymbol{e}_{(k)}^{i} \boldsymbol{e}_{j}^{(k)}=\delta_{j}^{i}\left(\delta_{i}^{i}=1, \delta_{j}^{i}=0\right.$ where $\left.i \neq j\right), a_{(k)}^{i j}=\boldsymbol{e}_{(k)}^{i} \boldsymbol{e}_{(k)}^{j}, \boldsymbol{m}^{(k)}$ are the unit vectors normal to the plane $\sigma_{(k)}$, satisfying the equations $\boldsymbol{e}_{i}^{(k)} \boldsymbol{m}^{(k)}=0, \boldsymbol{m}^{(k)} \boldsymbol{m}^{(k)}=1$.

According to the Kirchhoff-Love model, with an average bending for the functions $\psi^{(k)}$ and $\chi^{(k)}$ will take place following dependences.

$$
\psi^{(k)}=w_{, x}^{(k)}, \chi^{(k)}=w_{, y}^{(k)}
$$

where ()$_{, x}=\partial() / \partial x_{k}$. By using these relations for the covariant components of the tangential deformations are established following relations.

$$
\varepsilon_{i j}^{z_{(k)}}=\varepsilon_{i j}^{(k)}-z_{(k)} æ_{i j}^{(k)}
$$

where

$$
\begin{gathered}
\varepsilon_{11}^{(k)}=u_{, x}^{(k)}+\frac{1}{2} w_{, x}^{(k)} w_{, x}^{(k)}, \varepsilon_{22}^{(k)}=v_{, y}^{(k)}+\frac{1}{2} w_{, y}^{(k)} w_{, y}^{(k)} \\
2 \varepsilon_{12}^{(k)}=u_{, y}^{(k)}+v_{, x}^{(k)}+w_{, x}^{(k)} w_{, y}^{(k)} \\
æ_{11}^{(k)}=w_{, x x}^{(k)}, \quad æ_{22}^{(k)}=w_{, y y}^{(k)}, \quad æ_{12}^{(k)}=w_{, x y}^{(k)}
\end{gathered}
$$

Under the assumption of linear elastic behavior of core material when it is loading the contravariant components of the tension tensors in the walls $\sigma_{(k)}^{i j}$ with deformation tensor components $\varepsilon_{i j}^{z_{(k)}}$ are related by the elasticity correlation of the following form 


$$
\sigma_{(k)}^{i j}=\frac{E}{1-v^{2}} E_{(k)}^{i j m l} \varepsilon_{m l}^{z_{(k)}}
$$

where $E, v$ are the Young's modulus of elasticity and Poisson's ratio of wall material, and $E_{(k)}^{i j m l}$ is the tetravalent tensor of the elastic characteristics, components of which for an isotropic material in plane stress state are defined by correlations

$$
E_{(k)}^{i j m l}=v a_{(k)}^{i j} a_{(k)}^{m l}+\frac{1-v}{2}\left(a_{(k)}^{i m} a_{(k)}^{j l}+a_{(k)}^{i l} a_{(k)}^{j m}\right)
$$

Substitution of formulas (1.6) to the correlations (1.12) leads to the formulas

$$
\begin{aligned}
& E_{(k)}^{1111}=E_{(k)}^{2222}=\frac{1}{\sin ^{2} \varphi_{k}}, E_{(k)}^{1122}=E_{(k)}^{2211}=\frac{v \sin ^{2} \varphi_{k}+\cos ^{2} \varphi_{k}}{\sin ^{4} \varphi_{k}}, \\
& E_{(k)}^{1112}=E_{(k)}^{1121}=E_{(k)}^{1211}=E_{(k)}^{2111}=E_{(k)}^{1222}=E_{(k)}^{2122}=E_{(k)}^{2212}= \\
& =E_{(k)}^{2221}=-\frac{\cos \varphi_{k}}{\sin ^{4} \varphi_{k}}, \\
& E_{(k)}^{1212}=E_{(k)}^{2121}=E_{(k)}^{1221}=E_{(k)}^{2112}=\frac{1+\cos ^{2} \varphi_{k}-v \sin ^{2} \varphi_{k}}{2 \sin ^{4} \varphi_{k}}
\end{aligned}
$$

We assume that under the loading of the core in the sandwich and multilayer structural elements in its walls because of their small thickness realized the flat and membranaceous stress-strain state. In this case, accumulated in them potential energy of deformation is permissible to calculate by the following formulas

$$
\ddot{I}_{(k)}=\frac{1}{2} \int_{0}^{l_{k}} \int_{0}^{d} t\left(\sigma_{(k)}^{11} \varepsilon_{11}^{(k)}+2 \sigma_{(k)}^{12} \varepsilon_{12}^{(k)}+\sigma_{(k)}^{22} \varepsilon_{22}^{(k)}\right) \sin \varphi_{k} d x_{k} d y_{k}
$$

where $d \sigma_{k}=\sqrt{a_{k}} d x_{k} d y_{k}=\sin \varphi_{k} d x_{k} d y_{k}$ is the area of infinitely small element of the middle plane of $k$-th core wall, and the components of the deformation $\varepsilon_{i j}^{(k)}$ are defined by correlations (1.9). These correlations are written in linear approximation by dropping the nonlinear terms. After the transition from a flat membrane state in the perturbed moment state possible due to a loss of stability of the flat form of equilibrium. To study the perturbed neutral equilibrium state we will use the variational equation of the Ritz method, compiled on the basis of relations (1.8)-(1.11).

In the space $V$ of a selected cell of periodicity, referred to orthogonal Cartesian coordinates $x, y$ and $z$, averaged elastic properties of the core should be considered orthotropic. The elastic modulus of the first kind $E_{z}$ in the direction $z$ and the modules of the transverse shifts $G_{x z}, G_{y z}$ in the planes $x z$ and $y z$ must be determined within the bounds of the model of a transversely 
soft core. If the displacement vector of any point $M \in V$ provide an expansion $\boldsymbol{U}=u \boldsymbol{i}+v \boldsymbol{j}+w \boldsymbol{k}$ and in cross-sections $x=$ const,$y=$ const,$z=$ const introduce the averaged voltage $\tilde{\sigma}_{x z}, \tilde{\sigma}_{y z}, \tilde{\sigma}_{z}$ the accumulated in the volume strain energy will be determined by the expression

$$
\ddot{I}=\frac{1}{2} \iiint_{V}\left(\tilde{\sigma}_{x z} \gamma_{x z}+\tilde{\sigma}_{y z} \gamma_{y z}+\tilde{\sigma}_{z} \gamma_{z}\right) d V
$$

where for calculation the transverse shear strain $\gamma_{x z}, \gamma_{y z}$ and axial strain $\varepsilon_{z}$ of the homogenized medium in the linear approximation we have the kinematic relations

$$
\gamma_{x z}=\frac{\partial u}{\partial z}+\frac{\partial w}{\partial x}, \gamma_{y z}=\frac{\partial v}{\partial z}+\frac{\partial w}{\partial y}, \varepsilon_{z}=\frac{\partial w}{\partial z}
$$

Using of the principle of equivalence of real and replacing conditional core with average elastic and strength characteristics requires to preparation of the equality.

$$
\ddot{I}=\ddot{I}_{(1)}+\ddot{I}_{(2)}+\ddot{I}_{\left(1^{\prime}\right)}+\ddot{I}_{\left(2^{\prime}\right)}
$$

which will be used for further calculations.

\section{Critical values of the tensions formed in the walls of the core when it is loading}

Assume that in walls of the core in terms of loading formed homogeneous in their middle plane tensions $\sigma_{(k)}^{11}, \sigma_{(k)}^{22}$ and $\sigma_{(k)}^{12}$.

To produce the approximate structural formulas for using of theoretical and experimental method [8], the edges of the walls $x_{k}=0, x_{k}=l_{k}, y_{k}=0, y_{k}=d$ will be assumed simply supported.

Whether for these or other types of loading in the walls of the core forms only compressive tensions $\sigma_{(k)}^{11}$ and $\sigma_{(k)}^{22}$, and $\sigma_{(k)}^{12}=0$, when taken under considerationt pinning the edges of the walls to construct the solution of the stability function $w^{(k)}$ can be represented as

$$
w^{(k)}=A_{m n}^{(k)} \sin \frac{m \pi x_{k}}{l_{k}} \sin \frac{m \pi y_{k}}{d}
$$


where $m=1,2, \ldots, n=1,2, \ldots$ are the wave numbers. Then with the introduction of the dimensionless parameters

$$
\beta_{k}=\frac{l_{k}}{d}, \quad \alpha_{k}=\sigma_{(k)}^{22} / \sigma_{(k)}^{11} .
$$

For definition of critical value $\sigma_{(k)}^{11}$ it is possible to receive the formula

$$
\sigma_{(k)}^{11}=K_{x}^{(k)} \frac{D_{k}}{\sin ^{4} \varphi_{k}}=K_{x}^{(k)} D_{k}^{*}, \quad D_{k}=\frac{\pi^{2} E}{12\left(1-v^{2}\right)}\left(\frac{t}{l_{k}}\right)^{2}
$$

where

$$
K_{x}^{(k)}=\frac{\left(m^{2}+\beta_{k}^{2} n^{2}\right)^{2}+4 m^{2} n^{2} \beta_{k}^{2} \cos ^{2} \varphi_{k}}{m^{2}+\alpha_{k} \beta_{k}^{2} n^{2}}, \quad D_{k}^{*}=\frac{D_{k}}{\sin ^{4} \varphi_{k}}
$$

In general, the subcritical tension state, when $\sigma_{(k)}^{11} \neq 0, \sigma_{(k)}^{12} \neq 0$ and $\sigma_{(k)}^{22} \neq 0$ to construct the solution of the stability function $w^{(k)}$ can be represented as

$$
w^{(k)}=W^{(k)} \sin \frac{\pi y_{k}}{l_{k}} \sin \frac{\pi\left(y_{k}-k_{k} x_{k}\right)}{d}
$$

In a case $\sigma_{(k)}^{11}=\sigma_{(k)}^{22}=0$, using the (2.4) for determination the minimum critical tension $\sigma_{(k) *}^{12}=\tau_{\hat{e} \delta}$ it possible to receive an approximate formula

$$
\tau_{\hat{e} \delta}^{(k)}=\left(5.34+4 \frac{l_{k}^{2}}{d^{2}}\right) D_{k}^{*} \sqrt{1+\cos ^{2} \varphi_{k}}\left(1+\cos \varphi_{k} \sqrt{\frac{2}{1+\cos ^{2} \varphi_{k}}}\right)
$$

\section{Determination of elastic and strength characteristics}

Displacement vector in a space $V$ of core we can represent in the form

$$
\boldsymbol{U}=\frac{z}{h}\left(u_{0} \boldsymbol{i}+v_{0} \boldsymbol{j}+w_{0} \boldsymbol{k}\right), \quad 0 \leq z \leq h
$$

where $u_{0}=$ const, $v_{0}=$ const and $w_{0}=$ const - moving the points of the boundary plane $z=h$, with a net in-plane shear $x 0_{1} z\left(u_{0} \neq 0, v_{0}=0, w_{0}=0\right), y 0_{1} z\left(u_{0}=0, v_{0} \neq 0, w_{0}=0\right) \quad$ and tensioncompression in the direction of the axis $0_{1} z$. We denote

$$
\boldsymbol{U}_{0}^{(k)}=u_{0}^{(k)} \boldsymbol{e}_{(k)}^{1}+v_{0}^{(k)} \boldsymbol{e}_{(k)}^{2}+w_{0}^{(k)} \boldsymbol{m}^{(k)} \quad\left(k=1,2,1^{\prime}, 2^{\prime}\right)
$$


displacement vectors of faces $x_{1}=x_{1}^{\prime}=l_{1}$ and $x_{2}=x_{2}^{\prime}=0$ walls of the filler, and for the displacements $\boldsymbol{U}^{(k)}$ of the points $M_{k} \in \sigma_{(k)}$ we take the approximations

$$
\begin{aligned}
& \boldsymbol{U}^{(1)}=\frac{x_{1}}{l_{1}} \boldsymbol{U}_{0}^{(1)}, \boldsymbol{U}^{\left(1^{\prime}\right)}=\frac{x_{1}^{\prime}}{l_{1}} \boldsymbol{U}_{0}^{\left(1^{\prime}\right)}, \boldsymbol{U}^{(2)}=\left(1-\frac{x_{2}}{l_{2}}\right) \boldsymbol{U}_{0}^{(2)} \\
& \boldsymbol{U}^{\left(2^{\prime}\right)}=\left(1-\frac{x_{2}^{\prime}}{l_{2}}\right) \boldsymbol{U}_{0}^{\left(2^{\prime}\right)}
\end{aligned}
$$

The condition of equivalence of the real and the conventional core requires the satisfaction of the equality

$$
u_{0} \boldsymbol{i}+v_{0} \boldsymbol{j}+w_{0} k=\boldsymbol{U}_{0}^{(k)}=u_{0}^{(k)} \boldsymbol{e}_{(k)}^{1}+v_{0}^{(k)} \boldsymbol{e}_{(k)}^{2}+w_{0}^{(k)} \boldsymbol{m}^{(k)}\left(k=1,2,1^{\prime}, 2^{\prime}\right)
$$

Scalar multiplication of both sides by vectors $\boldsymbol{e}_{i}^{(k)}$ and using (1.2) (1.3), we arrive to the dependencies

$$
\begin{aligned}
& u_{0}^{(1)}=u_{0} \cos \alpha_{1}+w_{0} \sin \alpha_{1}, v_{0}^{(1)}=u_{0} \sin \psi+v_{0} \cos \psi \\
& u_{0}^{\left(1^{\prime}\right)}=u_{0} \cos \alpha_{1}+w_{0} \sin \alpha_{1}, v_{0}^{\left(1^{\prime}\right)}=u_{0} \sin \psi-v_{0} \cos \psi \\
& u_{0}^{(2)}=u_{0} \cos \alpha_{2}-w_{0} \sin \alpha_{2}, v_{0}^{(2)}=u_{0} \sin \psi+v_{0} \cos \psi \\
& u_{0}^{\left(2^{\prime}\right)}=u_{0} \cos \alpha_{2}-w_{0} \sin \alpha_{2}, v_{0}^{\left(2^{\prime}\right)}=u_{0} \sin \psi-v_{0} \cos \psi
\end{aligned}
$$

In component form the vector equalities (3.4) lead to the approximating functions

$$
\begin{aligned}
& u^{(1)}=\frac{x_{1}}{l_{1}} u_{0}^{(1)}, v^{(1)}=\frac{x_{1}}{l_{1}} v_{0}^{(1)}, u^{\left(1^{\prime}\right)}=\frac{x_{1}^{\prime}}{l_{1}} u_{0}^{\left(1^{\prime}\right)}, v^{\left(1^{\prime}\right)}=\frac{x_{1}^{\prime}}{l_{1}} v_{0}^{\left(1^{\prime}\right)} \\
& u^{(2)}=\left(1-\frac{x_{2}}{l_{2}}\right) u_{0}^{(2)}, v^{(2)}=\left(1-\frac{x_{2}}{l_{2}}\right) v_{0}^{(2)} \\
& u^{\left(2^{\prime}\right)}=\left(1-\frac{x_{2}^{\prime}}{l_{2}}\right) u_{0}^{\left(2^{\prime}\right)}, v^{\left(2^{\prime}\right)}=\left(1-\frac{x_{2}^{\prime}}{l_{2}}\right) v_{0}^{\left(2^{\prime}\right)}
\end{aligned}
$$

By the substitution into the linear kinematic relations

$$
\varepsilon_{11}^{(k)}=u_{, x}^{(k)}, \quad \varepsilon_{22}^{(k)}=v_{, y}^{(k)}, 2 \varepsilon_{12}^{(k)}=u_{, y}^{(k)}+v_{, x}^{(k)}
$$

compute the components of deformation in the walls of the core in the membrane approximation. 
Compiled on the basis of the relations, we consider the case of the periodicity cell of core by tension and compression along the axis $z\left(w_{0} \neq 0, u_{o}=v_{0}=0\right)$ in the case of loading we can get formulas:

$$
\begin{aligned}
& \varepsilon_{11}^{(k)}=\varepsilon_{11}^{\left(k^{\prime}\right)}=\sin ^{2} \alpha_{k} \frac{w_{0}}{h}, \varepsilon_{22}^{(k)}=\varepsilon_{22}^{\left(k^{\prime}\right)}=\varepsilon_{12}^{(k)}=\varepsilon_{12}^{\left(k^{\prime}\right)}=0 \\
& \sigma_{(k)}^{11}=\frac{E}{1-v^{2}} E_{(k)}^{1111} \varepsilon_{11}^{(k)}=\frac{E}{1-v^{2}} \frac{\sin ^{2} \alpha_{k}}{\sin ^{4} \varphi_{k}} \frac{w_{0}}{h} \\
& \sigma_{(k)}^{22}=\frac{E}{1-v^{2}} E_{(k)}^{2211} \varepsilon_{11}^{(k)}=\left(v \sin ^{2} \varphi_{k}+\cos ^{2} \varphi_{k}\right) \sigma_{(k)}^{11} \\
& \sigma_{(k)}^{12}=\frac{2 E}{1-v^{2}} E_{(k)}^{1211} \varepsilon_{11}^{(k)}=-\cos \varphi_{k} \sigma_{(k)}^{11}
\end{aligned}
$$

From which follows that in the condition of tensile fracture of core $\left(w_{0}>0\right)$ is possible because of the destruction of the adhesive layer connecting the core with a bearing layer, and while compression $\left(w_{0}<0\right)$ due to loss of stability of the walls in her bilateral compression and shear.

When we approximating the displacements of the averaged core (3.1), it formed the strains and stresses

$$
\begin{aligned}
& \varepsilon_{z}=\frac{w_{0}}{h}, \gamma_{x z}=\frac{u_{0}}{h}, \gamma_{y z}=\frac{v_{0}}{h}, \tilde{\sigma}^{33}=E_{z} \frac{w_{0}}{h} \\
& \tilde{\sigma}^{13}=G_{x z} \frac{u_{0}}{h}, \tilde{\sigma}^{23}=G_{y z} \frac{v_{0}}{h}
\end{aligned}
$$

where the homogenized elastic modules $E_{z}, G_{x z}$ and $G_{y z}$ must be determined. By using the relations (3.10) in accordance with expression (1.15) for calculation $\ddot{I}$ we arrive at the formulas in the case $u_{0}=v_{0}=0$

$$
\begin{aligned}
& \ddot{I}=h d\left(\operatorname{ctg} \alpha_{1}+\operatorname{ctg} \alpha_{2}\right) \sin \psi \tilde{\sigma}^{33} w_{0} \\
& \ddot{I}=d\left(\operatorname{ctg} \alpha_{1}+\operatorname{ctg} \alpha_{2}\right) \sin \psi E_{z} w_{0}^{2}
\end{aligned}
$$

and by using relations (3.8) and (3.9), in accordance with (1.14) for calculation $\ddot{I}_{(k)}$ we arrive to the formulas

$$
\begin{aligned}
& \ddot{I}_{(k)}=\sigma_{(k)}^{11} t d \frac{\sin \alpha_{k} \sin \varphi_{k}}{2} w_{0}\left(k=1,2,1^{\prime}, 2^{\prime}\right) \\
& \ddot{I}_{(k)}=\frac{E}{1-v^{2}} t d \frac{\sin ^{3} \alpha_{k}}{2 \sin ^{3} \varphi_{k}} \frac{w_{0}^{2}}{h}\left(k=1,2,1^{\prime}, 2^{\prime}\right)
\end{aligned}
$$


By substituting relations $(3.12)$, (3.14) from equation (1.17) we can obtain a formula to determine $E_{z}$

$$
E_{z}=\frac{E}{1-v^{2}} \frac{t}{h\left(\operatorname{ctg} \alpha_{1}+\operatorname{ctg} \alpha_{2}\right)} \sum_{k=1}^{2} \frac{\sin ^{3} \alpha_{k}}{\sin ^{3} \varphi_{k}}
$$

and using (3.13) from (1.17) we can obtain the following dependence

$$
\tilde{\sigma}^{33}=\frac{t}{h \sin \psi\left(\operatorname{ctg} \alpha_{1}+\operatorname{ctg} \alpha_{2}\right)} \sum_{k=1}^{2} \sigma_{(k)}^{11} \sin \alpha_{k} \sin \varphi_{k}
$$

In the case of the maximum tensile stresses $\sigma_{(k)}^{11}, \sigma_{(k)}^{22}, \sigma_{(k)}^{12}$ are largest in the wall, in which the maximum value is $\theta_{k}=\sin ^{2} \alpha_{k} / \sin ^{4} \varphi_{k}$. The process of destruction in it begins when certain tensile strength $\sigma^{+}$is reached. As in real cores, angles $\alpha_{k}$ and $\varphi_{k}$ do not have significant difference, it is possible to assume that when the strength reaches $\sigma_{(1)}^{11}$ or $\sigma_{(2)}^{11}$ the simultaneous failure of all four walls of the core begins. Under these assumptions to determine the limit $\tilde{\sigma}_{+}^{33}$ at which the core is destroyed we can create equality

$$
\tilde{\sigma}_{33}^{+}=\frac{\operatorname{t\sigma ^{+}}}{h \sin \psi\left(\operatorname{ctg} \alpha_{1}+\operatorname{ctg} \alpha_{2}\right)} \sum_{k=1}^{2} \sin \alpha_{k} \sin \varphi_{k}
$$

From it by the experimentally determined value $\tilde{\sigma}_{+}^{33}$ determined the quantity $\sigma^{+}$.

When compressing of the core begins its destruction due to the destruction of the buckling of the wall, which formed the voltage $\sigma_{(k)}^{11}$ at a given dimensions

$$
\mu_{k}=v \sin ^{2} \varphi_{k}+\cos ^{2} \varphi_{k} \text { and } \cos \varphi_{k}=-\sigma_{(k)}^{12} / \sigma_{(k)}^{11}
$$

reaches a critical value

$$
\sigma_{(k) *}^{11}=K_{x}^{(k)} \frac{\pi^{2} E}{12\left(1-v^{2}\right) \sin ^{4} \varphi_{k}}\left(\frac{t}{l_{k}}\right)^{2}=K_{x}^{(k)} \frac{\pi^{2} E \sin ^{2} \alpha_{k}}{12\left(1-v^{2}\right) \sin ^{4} \varphi_{k}}\left(\frac{t}{h}\right)^{2}
$$

Assuming further that for the real core loss of stability of a wall immediately causes a loss of stability of a second wall, we form the equation $\sigma_{(k) *}^{11}=\sigma_{(k)}^{11}$, from which to determine the lateral deformation at the moment of loss of stability [9] by using relations (3.9) and (3.18) follows the formula

$$
\left(\frac{w_{0}}{h}\right)^{*}=K_{x} \frac{\pi^{2}}{12}\left(\frac{t}{h}\right)^{2}
$$


where $K_{x}^{(1)} \approx K_{x}^{(2)} \approx K_{x}$. However

$$
\tilde{\sigma}_{*}^{33}=K_{x} \frac{\pi^{2} E}{12\left(1-v^{2}\right) \sin \psi\left(\operatorname{ctg} \alpha_{1}+\operatorname{ctg} \alpha_{2}\right)}\left(\frac{t}{h}\right)^{3} \sum_{k=1}^{2} \frac{\sin ^{3} \alpha_{k}}{\sin ^{3} \varphi_{k}}
$$

For given parameters $E, v, t / h, \alpha_{k}$ and $\varphi_{k}$ of the core the experimentally determined value of critical stress $\tilde{\sigma}_{-}^{33}$ of the derived formula (3.20) is determined by a dimensionless coefficient $K_{x}$.

Elastic and strength characteristics of the core in a shift in planes $\mathrm{XoZ} \mathrm{YoZ}$ are defined by analogy with the stated above.

\section{Conclusions}

Theoretical and the experimental methods presented in this article allow to define the averaged elastic and strength characteristics of sandwich folded fillers on the basis of use of analytical expressions for definition of the critical tensions formed in walls of a filler while loading.

\section{References}

[1] Noor A. K., Burton W.S., Bert Ch. W., Computational models for sandwich panels and shells. Applied Mechanics Reviews, 1996, V. 49, ${ }^{1}$ 3, p. 155-199.

[2] Bersudsky V.E., Krisin V.N., Forest S., Manufacturing technology of cellular aircraft structures. - Moscow: Mashinostroenie, 1975. 216s.

[3] Aleksandrov A.Y., Bryukker L.E., Kurshin L.M., The calculation of sandwich panels / M. Oborongiz, 1960.272s.

[4] Panin V.F., Gladkov Yu.A., Constructions with cores. Directory. Moscow: Mashinostroenie. 1991. 272s.

[5] Kryutchenko V.E., Analysis of optimum insulation properties of sandwich plates with honeycomb core. /Mechanics of Composite Materials. - 1993, T.29.- № 6.- S.835-839.

[6] Relsey S., Gellatly H. and Clark B., The shear modulus of foil honeycomb cores. Aircraft Engineering. 1958. V.30. № 356. P.294-302.

[7] Bakhvalov N.S., Ponasenko G.P., Averaging of the processes in periodic media.- Moscow: Nauka, 1984. $352 \mathrm{c}$.

[8] Sachenkov A.V., Theoretical and experimental method for studying the stability of plates and shells. Investigations on the theory of plates and shells. Kazan: Izdatel'stvo Kazan. State. Univ. - 1970, Vol. 6.7.- S. 391433.

[9] Volmir AS Stability of elastic systems. M.: Gos. out of Sci. literature. 1963. $880 \mathrm{sec}$. 
[10] Akishev N.I., Zakirov I.I., Paimushin V.N., Shishov M.A., Theoreticalexperimental method for determining the averaged elastic and strength characteristics of honeycomb of sandwich structures. Mechanics of Composite Materials. - 2011 (in press).

[11] Zakirov I.M., Alekseev, K.A., Akishev N.I. Kayumov R.A., NikitinA.V., Zakirov I.I., Manufacturing of sandwich panels with folded core of polymer paper Kazan: Publishing house «Fan» 2009

[12] Zakirov I.M., Alekseev K.A., Determining of the parameters of the four radial helical folded structure / IVUZ "Aviatcionnaya technika" 2005 